Reaktion nur auf Wasserentziebung und vielleicht anf Oxydation beruben. Andere wasserentziehende und oxydirende Mittel wie Schwefelsăure, Chlorzink, Arsensăure, Nitrobenzol gaben beim Schmelzen mit Pyrogallusgăure wohl rothbraun bis schwarz aussehende farbatoffăhnliche Substanzen, aber kein Gallein. Hingegen scheint die Gallergthrosăure von Wacken roder und die Blangallussäure von Berzelius, welche dorch Bebandlung der Gallnssäure mit Alkalien erbalten worden, nacb der allerdings nur oberfilichlichen Beschreibung mit dem Gallein identisch zu sein.

Die weitere Untersuchang des Galleins und die Verfolgung der beschriebenen Resktion behalte ich mir vor.

197. V. จ. Richter: Ontersuchungen übor die Constitution der Benzolderivate.

(Eingegangen am 14. Mai.)

Die Isomerieverbăltnisse der Benzolderivate sind von wesentlichem Interesse fur die chemische Constitutionstheorie, da aus denselben wsbrscheinlich neue Gesichtopunkte über die Lagerung der Atome sich werden ableiten lassen. Obgleich in letzter Zeit Gegenstand mehrfacher Untersuchungen, sind dieselben jedoch noch lange nicht definitiv festgestellt. Unsere beutige Theorie der Benzolverbindungen beruht auf der symmetrischen Constitution des Benzols, dargestellt durch das regelmäвsige Sechseck; die factische Grundlage dazu besteht in der Existenz dreier isomeren Reiben für die Derivate mit zwei substituirenden Gruppen. A uf Grundlage der Sechseck-Theorie ergiebt sich gun die Aufgabe, für alle Benzolderivate (zunächst für die mit zwei Grappen) die Stellung der Gruppen im Benzolkern nachzuweisen.

Es scheint mir, dass diese Anfgabe häufig etwas voreilig entschleden worden ist. So wird gewöbnlich sngenommen, häufig sogar als bowiesen betrachtet, dass im Chinon die beiden Sauerstoffatonic benachbarte Stellungen einnebmen; diese Annabme ist jedoch ron C. Graube offenbar nur aus geometriachen Rücksichten gemacht worden, - ein Beweis hierfür existirt durchaus nìcht.

Als einzige Grundlage zur Bestimmung der chemischen Orte im Benzol dienen bis jetzt die drei Phtalsăuren. Um daher die Constitution der Benzolderivate zunächst mit zwei Gruppen zu bestimmen, muss man für dieselben einen directen oder indirecten Uebergang zu einer der Phtalsăuren nacbweisen. Jedoch darf ein derartiger Uebergang noch nicht als entucheidend angesehen werden, ds bei den mannigfaltigen Reactionen eine Umsetzang eines Körpers in den einer andern Roibe stattinden kann. Dass derartige Umsetsungen, welche in der Fettgrappe bänfiger beobachtet sind, auch in der Benzolgrappe 
stattfinden, ist zunächst von Kekalé an der Metaphenolsulfosăure nachgewiesen worden. Ich werde im Folgenden zeigen, dass dieselben băufiger vorkommen, - zunk̈chst an dem Dibrombenzol.

Ueber das Dibrombenzol. V. Meyer hat (diese Ber. III. S. 754) aus dem durch directes Bromiren von Benzol gewonnenen Dibrombenzol, welches bei $89^{\circ}$ schmilzt, Terephtalsäure erhaltea. Er schliesst daraus, dass dieses Dibrombenzol zar Parareihe gehöre. Andererseits wird das Dibrombenzol durch Einwirkang von Phosphorpentabromid auf Bromphenol erhalten (A ug. Mayer), ans welcbem Kōrner (Ann. Chem. Pharm. 137, S. 221) gewöhnliche Oxybenzoẽsãure dargestellt hat. Darbach gehörte das Dibrombenzol zur Orthoreihe. Obgleich die von V. Meger für diese Verbăltnisse gegebene Erklärung sich auf einem analogen Fall stützt, schien mir dieselbe doch für den gegebenen Full etwas unwabrscheinlich zu sein, zumal da das entsprechende Chlorphenol, mehreren Uebergängen zu Folge, zur Reihe des Chinons gehört. Ich habe in der That gefunden, dass das Dibrombenzol einerseits zum Chinon, andererseits zur Reihe der Orthooxybenzoësäure gehört.

Bromnitrobenzol, durch Nitriren von Brombenzol erhalten und bei $125^{\circ}$ schmelzend, wurde vermittelst $Z$ inn und Salzsāure in Bromamidobenzol verwandelt, welches, mit Wasser überdestillirt, bei $61-62^{\circ}$ schmolz und in Octaedern krystallisirte. Das salpetersaure Salz krystallisirt in langen, geraden Nadeln, die in warmem Wasser leicht, in kaltem schwer löslich sind. Dasselbe wurde mit salpetriger Sănre behandelt und die Lösung mit Bromwasser gefällt. Das orhaltene Diazoperbromid wurde mit kochendew Alkohol (98 pCt.) zersetzt. Beim Erkalten erstarrte die Lösnng zu einem Brei ans grossen glánzenden, zerbrechlichen Blättchen. Dieselţen schmolzen bei $89^{\circ} \mathrm{C}$. und zeigten ganz den Habitus des Dibrombenzols aus Benzol, welches zam Vergleiche dargestellt wurde.

Es gehört demnach das bei $89^{\circ}$ schmelzende Dibrombenzol zar Reihe des Chinons, da das Bromnitrobenzol, aus welchem es exhalten wurde, ebenfalls aus Nitranilio entsteht (Griess, Jahrb. 1863); letzteres giebt aber nach Hofmann durch Reduction und Oxydation Chinon. Da ein solcher Uebergang rom Bromnitrobenzol zum Chinon ziemlich entfernt ist, stellte ich einen näheren dar. Das Bromnitrobenzol wurde mit Kalilauge in zugeschmolzener Röhre erhitzt und dabei Orthonitrophenol erbalten, welches aus Wasser in langen charakteristischen Nadeln krystallisirte, die bei $108^{\circ} \mathrm{C}$. (anstatt $110^{\circ}$ ) schmolzen. Das Orthonitrophenol giebt aber nach Körner Amidophenol und Chinon.

Nach zwei Uebergängen gehört demnach das Dibrombenzol zur Reihe des Chinons, und nehme ich bei dem Uebergange desselben in Terephtalsäure eine Umsetzung an, über welche ich später sprechen 
werde. Man sieht hieraus jedenfalls, dass ein Uebergang für die Constitution nicht entscheidend ist. Theoretisch dürfte man nur dann sicher gehen, wenn von drei isomeren Körpern drei analoge Uebergänge vorliegen. Von diesem Grundsatze auggehend unternahm jch es, von den drei Brom- und Chlornitrobenzolen zu den entsprechenden substituirten Benzoësäuren zn gelangen, da der Zusammenhang zwischen den Benzol- und Phenolderivaten und den substituirten Toluolen und Benzoësäuren noch sebr unsicher war. Nach vielen vergeblichen Versuchen gelang es mir hierbei eine Reaction zu finden, dnrch welcbe diese Aufgabe wesentlich gefördert wurde.

Ueber eine neue Synthese pon Sanren. Es besteht diese Resction, wie ich schon in einer vorls̈ufigen Notiz mitgetheilt, in der Einwirkung ron Cyankalium anf gewisse Nitrokörper, nach der Gleichung

$$
\mathrm{C}^{6} \mathrm{H}^{4}\left\{\begin{array}{l}
\mathrm{Br} \\
\mathrm{NO}^{2}
\end{array}+\mathrm{CNK}=\mathrm{C}^{6} \mathrm{H}^{4}\left\{\begin{array}{l}
\mathrm{Br} \\
\mathrm{CN}
\end{array}+\mathrm{KNO}^{2} .\right.\right.
$$

Ich erbitze die Nitrokörper mit ungefăhr 2 Aeq. Cyankalium (reinem) und Alkohol in zugeschmolzenen Köbren auf $180^{\circ}-200^{\circ} \mathrm{C}$. und hōher, während 3-5 Stundea. Wenn die Reaction stattgefunden bat, findet sich in der Röhre koblensaures Ammoniak sublimirt, und der dunkle Inbalt ist zu einem dicken Brei erstarrt. Beim Oeffnen der Röhre entweicht viel Ammoniak. Im Inbalte lässt sich (bei stattgefundener Reaction) viel satpetrige Säure nachweisen. Derselbe wird alsdann mit Alkohol und Kalihydrat gekocht, so lange nocb Ammoniak entweicht, wit Wasser versetzt, filtrirt, durch Salzsäure gefällt, in kohlensaurem Ammoniak gelöst und mit Tbierkoble gekocht. Dieser Reinigangsprocess wird mehrere Male wiederholt, bis die gefällte Säure weisa ist; das kohlensaure Ammoniak lässt bierbei stets etwas braune Substanz ungelöst. Die Ausbeute an Säure ist sehr reichlich - bis zu $40 \mathrm{pCt}$. der theoretischen Menge. Es unterliegen dieser Reaction, soweit bis jetzt untersucht, zwei der drei isomeren Chlor- und Bromnitrcbenzole, so wie angenscheinlich auch die sogenannten salpetrigsavr 3 Aether, über welche später.

Die isomeren Bromnitrobenzole. Durch Nitriren von Brciabenzol (bei $153-155^{\circ}$ siedend) entsteher, wie Hübner und Alsberg gezeigt (Ann. Chem. Pharm. Bd. 156, S. 311), zwei Nitrobrorsbenzole; das eine, bei $125^{\circ}$ schmelzend, wird leicht rein erbalten, das andere, aus der Mutterlauge durch Krystallisation abgeschieden, bild'st längere dicke Nadeln und schmola bei $36^{\circ}$ und $39^{\circ} \mathrm{C}$.; es gelang nicht dasselbe ganz frei ron dem ersteren zu erbalten. Bei der Destillation mit Wasser geht das erstere leichter über. Das dritte Bronnitrobenzol wurde nach Griess aus dem Dinitrobenzol durgestel i Das salpetersaure Salz des sogenannten Paranitranilins in die Diazoverbindung übergeführt, mit Bromwasser gefällt, das Perbromid 
nit Alkobol zersetzt und die Masse mit Wasser destillirt, giebt bei $56^{\circ}$ C. schmelzendes Bromnitrobenzol.

Uebergang vonden Bromitrobenzolen zu den Brombenzoesăuren. 1) Das bei $125^{\circ}$ schmelzende Bromnitrobenzol giebt mit Cyankalium auf $180^{\circ}$ erbitzt leicht uad reichlich Orthobrombenzoёвäure. Das Ammoniaksalz der rohen Săure wurde in coucentrirter Lösung durch Chlorbaryum gefällt. Die aus dem Baryumsalz abgeschiedene Săure schmolz bei $152^{\circ}$. Aus der vom Baryumsalz abfiltrirten Lösung wurde eine geringe Menge Săure erbalten, die gegen $150^{\circ}$ schmolz. Ans einer anderen Bereitung schmolz die Săure bei $150^{\circ}$; sublimirt schmolzen beide bei $153^{\circ}$. $0.1514 \mathrm{Grm}$. Substanz gaben $0.1423 \mathrm{Grm} . \mathrm{Ag} \mathrm{Br}=40.0 \mathrm{pCt}$. Brom; $\mathrm{C}^{7} \mathrm{H}^{5} \mathrm{BrO}^{2}$ verlangt 39.9 pCt. Durch Schmelzen der Säure mit Kalibydrat warde Orthooxybenzoësäure erhalten, die aus $W$ asser in warzigen, bei $190^{\circ}$ schmelzenden Krusten krystallisirte.

2) Das bei $56^{\circ}$ schmelzende Bromnitrobenzol aus Dinitrobenzol giebt ebenfalls mit Cyankalium lejcht eine Bromsaure. Das A mmoniaksalz der roben Săure wurde mit Chlorbaryum versetzt. Nach dem Einengen und Erkalten schied sich eine geringe Menge eines Baryumsalzes sus. Aus der abfiltrirten Löøung warde durch Salseăure eine Säurc gefällt, die bei $137^{\circ}$ schmolz; mit Wasser gekocht schmolz der gelöste Theil bei $137^{\circ}$, der ungelöste bei $137.5^{\circ} \mathrm{C}$. Sublimirt schmolz die Săare bei $137.5^{\circ} \mathrm{C}$. $0.1952 \mathrm{Grm}$. der Share gaben $0.1838 \mathrm{Grm}$. $\mathrm{AgBr}=40.0 \mathrm{pCt}$. $\mathrm{Br}$ - die Formel $\mathrm{C}^{7} \mathrm{H}^{5} \mathrm{BrO}^{2}$ verlangt $39.9 \mathrm{pCt}$. Beim Schmelzen der Säure mit Kalibydrat wurde Salicylsäure erbalten, die in den charakteristiselien Nadeln krystallisirte. Es ist demnach diese Säure Metabrombenzoësăure. Eine nähere Beschreibung dieser Säure, die ich auch aus Authranileänre erhalten, folgt weiter; sie charakterisirt sich durch die Löslichkeit ihres Baryumsalzes. Aus der kleinen Menge Baryumsalzes, das sich ausgeschieden (siehe oben), wurde eine brombaltige Säure erhalten, die sublimirt bei $243^{\circ}$ schmolz, - slso wahrscheinlich Parabrombenzoësäure war. Während von letzterer sich nur wenig bildet, entsteht die Metabrombenzoësăure in reichlicher Menge, gegen $40 \mathrm{pCt}$. vom Bromnitrobenzol. Icb nehme an, dass bei der hohen Tenperatur der Reaction (200 $\mathrm{C}$.) eine geringe Umsetzung stattindet. Ein gleiches Auftreten von Parasänre neben Metabrombenzoësäure habe ich auch bei der Bildung der hetzteren aus Anthranilsäure beobachtet.

3) Das dritte Bromnitrobenzol (aus Brombenzol), welches bei $37^{\circ}$ C. scbmolz, gab mit Cyankalium auf $200^{\circ}$ erhitzt nur eine Spur von Orthobrombenzoësäure. Ich schloss daraus auf eine Verunreinigung derselben mit bei $225^{\circ}$ schmelzendem Orthobromnitrobenzol. Es gelang in der That letzteres in dem angewandten Bromnitrobenzol (bei $37^{\circ}$ schmelzend) durch Krystallisation Alkohol nachzuweisen. 
Das umkrystallisirte Bromnitrobenzol scbmolz bei $38-39^{\circ}$ C., gab aber mit Cyankalium abermals eine Spur Ortbobrombenzoěsara, die bei $153^{\circ}$ schmolz. Beim Erhitzen desselben nuf $280^{\circ} \mathrm{C}$, wobei schon Verkohlung stattfand, wurde dasselbe Resultat erhalten. Ich schliesse daraus, das das bei $37-39^{\circ}$ schmelzebde. Bromnitrobenzol, ans welchem nach den beiden vorbergebenden Uebergängen Parabrombenzoz. săure zu erwarten war, mit Cyankalium nicht zu reagiren vermag. Nimnit man an, dass die relative Stellung des Broms zur Nitrogruppe auf die Reactionsfähigkeit der letzteren influirt, so erscheint es begreiflich, dass bei der Parastellung keine Reaction stattindet.

Da also der dritte Uebergang nicht gelingt, der Beweis für die Constitution des dritten Bromnitrobenzols also ein indirecter ist, wiederholte ich diese Reactionen mit den Chlornitrobenzolen.

Die isomeren Chlornitrobenzole. Durch Nitriren von Chlorbenzol entstehen zwei Chlornitrobenzole: das eine kryatallisirte ans Alkohol in langen Prismen, die bei $84^{\circ}$ schmolzen - das andere ist flüssig. Das erstere wird ebenfalls aus Nitranilin ( $G$ rie s8) und durch Einwirkung ron Phosphorpentachlorid auf Orthonitrophenol erhalten. Ich trennte beide durch Destillation mit Wasser, wobei von dem Güssigen nur sebr wenig erhalten wurde. Das dritte Chlornitrobenzol wurde nach Griess aus Paranitranilin (aus Dinitrobenzol) erbalten. Die sslpetersaure Diszoverbindung wurde mit Platinchlorid und Alkobol gefallt, das Platindoppelsalz mit Chlornatrium und Soda destillic. Die Ausheute mit Chlornatrium ist reicblicher, aber weniger rein. Das erhaltene Chlornitrobenzol wurde mit Wasser überdestillirt und schmolz bei $46^{\circ} \mathrm{C}$. Die gescbmolzene Masse krystallisirt in dioken Prismen.

Uebergang von den Chlornitrobenzolenzuden Chlorbenzoësäuren. 1) Das bei $84^{\circ}$ schmelzende Chlornitrobenzol giebt mit Cyankalium auf $200^{\circ}$ erhitzt Orthochlorbenzoẽ äure. Das Ammoniaksalz der erhaltenen Säure wird durch Chlorcalcjum gefällt. Das scliwer lösliche Chlorcalciumsalz krystallisirte in concentriech gruppirten dicken Nadeln. Die aus dem Calciumsalz gewonnene Säure scbmolz bei $152^{\circ}$ - die Säure aus der abfiltrirten Lögung ebenfalls bei $152^{\circ}$. Sie sublinirte in isolirten geraden Nadeln, die bei $153^{\circ}$ schmolzen. $0.1855 \mathrm{Grm}$. gaben $0.1645 \mathrm{Grm} . \mathrm{AgCl}=22.2 \mathrm{pCt}$. $\mathrm{Cl}$; die Formel $\mathrm{C}^{7} \mathrm{H}^{5} \mathrm{ClO}^{2}$ verlangt $22.6 \mathrm{pCt}$. Cl. Beim Schmelzen der Säure mit Kalihydrat wurde Orthonxybenzoësäure erbalten.

2) Das bei $46^{\circ}$ schmelzende Chlornitrobenzol reagirt mit Cyankalium nicht bei $200^{\circ}$, wohl aber bei $250-270^{\circ}$. Das Ammoniaksalz der erhaltenen Säure wird nicht durch Chlorcalcium gefällt. Die ausgeschiedene Säure schmolz sublimirt bei $132^{\circ}$ und $134^{\circ}$. Die aus der Mutterlauge erhaltene Säure schmolz bei $137^{\circ}$. Sie sublimirt in glänzenden Flocken, wälırend die Orthochlorbenzoësäure isolirte gerade Nadeln giebt. Beim Schmelzen mit Kalihydrat wurde eine Săure erhalten, 
die mit Eisenchlorid tief violett färbte - also Salicylsänre. Darch den Schmelzpunkt and die grosse Löslichkeit des Calciumsalzes wird die erhaltene Ssure als Chloraalylsă a re charałterisirt.

Das dritte flüssige Chlornitrobenzol wurde nicht untersucht, da keine Reaction zu erwarten war. Ich beabsichtige in derselben Weise die Jodnitrobenzole za untersuchen, aus dem Dinitrobenzol das dritte Jodnitrobenzol, und daraug die Jodsalylsäure zu erhalten.

Ich habe also aus dem bei $125^{\circ}$ chmelzenden Bromnitrobenzol Orthonitrophenol und Orthobrombenzoësäure erhalten, aus dem Orthochlornitrobenzol: Orthochlorbenzoësäure. Es gehört demnach das Chinon zur Reihe der Orthoderivate der Benzoësăıre. Wenn nun die letzteren nach V. Meyer die Stellung $(1,3)$ baben, so gilt dasselbe für das Cbinon. Ferner wurde ans dem Dinitrobenzol Paranitranilin, die Brom- und Chlornitrobenzole und daraus Metabrom- und Metachlorbenzoēsäure erbalten: es gehören danach die sogenaunten Paraderivate des Benzols und Phenols, das Parajodanilin, das Resorcin zur Reibe der Salicylgăure. Hieraus folgt, dass die dritte isomere Reihe, das flüssige Chlornitrobenzol, das bei $37-39^{\circ}$ schmelzende Bromnitrobenzol, das flüchtige Nitrophenol, das Pyrocatechin zur Parareibe gehören :

$\begin{array}{lrcc}\mathrm{C}^{6} \mathrm{H}^{4} \mathrm{BrN} \mathrm{O} \mathrm{O}^{2} & 125^{\circ} & 56^{0} & 37^{0} \\ \mathrm{C}^{6} \mathrm{H}^{4} \mathrm{Cl} \mathrm{N} \mathrm{O} \mathrm{O}^{2} & 84 & 46 & \text { 6üssig } \\ \mathrm{C}^{7} \mathrm{H}^{5} \mathrm{BrO}^{2} & 153 & 137.5 & - \\ \mathrm{C}^{7} \mathrm{H}^{5} \mathrm{Cl} \mathrm{O}^{2} & 152 & 137 & -\end{array}$

Nan gaben aber nach den Untersuchungen von Irelan und Garrick die Brombenzolsulfosäure und die Benzoldisulfosäure durcb Destillation mit Cyankalium Terephtalsüure, andererseits gaben beide Skauren beim Schmelzen mit Kalihydrat Resorcin. Darnach gebörten die sogenannten Paraderivate des Phenols zur Reihe der Terephtalsăure. Ich nehme an, dass beim Scbrnelzen der beiden Säuren mit Cyankalium, wobei Terephtalsäure entsteht, eine Umlagerung stattfindet, indem dio zwei Cyangruppen sich von einander entfernen. Ein analoges Verbalten zeigen das Cblorätbylen und Chloräthyliden, aus welchem letzteren, ebenso wie aus ersterem, vermittelst Cyankslium Aethylenbernsteinsăure erbalten wird. Es gelingt nicht mebr als ein $C N$ an ein Koblenstoffatom zu binden, zwei CN-Gruppen scheinen sich in der Nähe zu hindern. In ähnlicher Weise liesse sich die Bildung der Terephtalsäure (nach V. Meyer) aus Dibrombenzol erklären, welches, wie gezeigt, zur Cbinonreibe gehört. Hieraus erklären sich auch die vergeblichen Versuche aus dem Orthobromtoluol das entsprechende Xylol zu erhaiten, in der Orthobrom- und Orthonitrobenzoësäure (siebe später) $\mathrm{Br}$ und $\mathrm{NO}^{2}$ durch $\mathrm{CH}^{3}$ und $\mathrm{CN}$ 2u ersetzen. Um 
definitiv meine Uebergänge und diese Ansichten zu bestätigen, muss ich einen Uebergang von der dritten isomeren Reihe des Benzols zur 'Terephtalsäure nachweisen. Ich habe dazu begonnen, von dem flüchtigen Nitrophenol ausgehend, in dem daraus erhaltenen Jodphenol Carboxyl zu substituiren. Falls bierbei Paraoxybenzoësäure entstände, wären die gegebenen Uebergänge bewiesen; andernfalls wäre die Reaction mit allen drei substituirten Phenolen durchzumachen.

Ich enthalte mich aller Betrachtung: $n$ über den gegenseitigen Einfluss der substituirenden Gruppen, so lange die Structur der Ortho- und Metaderivate der Benzoěsāure noch nicht mit Sicherheit bestimmt ist. Die beiden Uebergänge von V. Meyer zur Isopbtalsäure scheinen mir noch nicht definitiv entscheidend, da beide zu einer Reibe gehören. Um diese Erage zu entscheiden, babe ich versucbt, aus dem Nitrobenzošsäureăther vermittelst Cyankalium die entsprechende Phtalsäure zu erbalten. Die Reaction findet hierbei aber nicht statt, was aus dem Vorhergehenden begreiflich. Ich beabsichtige in derselben Weise die beiden Bromnitrobenzoësäuren zu untersuchen.

Zur Bestätigung der von ihm angenommenen Stellungen hat V. Meyer die Schmelzpunkte der Phtalsänren herangezogen*); die Schmolıpunkte der Toluylsäuren jedoch widersprecben dem:

$\begin{array}{lllrlc}\text { Phtalsäure } & 185^{\circ} & \text { Isophtalsäure } & 300^{\circ} & \text { Terephtslsäure } & - \\ \text { Toluylsäure } & 102^{\circ} & \text { Isotoluylsäure } & 90^{\circ} & \text { Paratoluylsäure } & 176^{\circ}\end{array}$

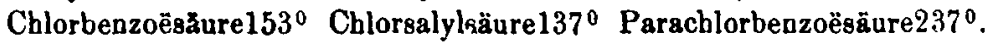

Es scheint mir aber wahrscheinlicher, dass die Schmelzpunkte der substituirten Benzoësäuren eher den Toluylsäuren folgen werdeo, da in beiden zwei rerschiedene Gruppen sich befinden. Bei Derivaten mit zwei gleichen Gruppen scheinen sich die Schmelzpunkte anders zu verhalten, wie an den Dioxybenzolen zu sehen.

\section{Ueber die Metabrombenzoēsäure.}

Wie im Vorhergehenden gezeigt, babe ich aus dem bei $56^{\circ}$ schmelzenden Bromnitrobonzol vermittelst Cyankalium eine Brombenzoësäure erhalten, die bei $137.5^{\circ}$ schmilzt und mit Kalihydrat geschmolzen Salicylsäure giebt. Dieselbe Säure babe ich auch aus der Anthranilsäure erhalten. Das salpetersaure Salz der Anthranilsäure wurde mit Wasser übergossen, mit salpetriger Saure bis zur Lösung behandelt, und mit Bromwasser gefällt. Das Diazoperbromid scheidet sich in rothen, barten Krystallen aus, die ziemlich löslich sind, so dass aus der abfiltrirten Lösung beim Stehen in der Kälte sich noch ziemliche Mengen des Perbromides ausscheiden. Das Perbromid wurde mit Alkohol ( 88 und $96 \mathrm{pCt}$.) übergossen und bei gelinder Wärme zersetzt, verdunstet, der Rückstand mit alkoholischem Kali gekocht

* Ann. Chem. Pharm. 156, S. 280. 
zur Zersetzung des gebildeten Aethers der Brombenzoēsăure, darauf mit Såare gefăllt und in kohlensaurem Ammoniak gelöst. $E_{8}$ bleibt bierbei ziemlich viel eines braunen krystallinischen (Bromanilartigen) Körpers ungelöst. Die Lösung wird zur Entfärbung mebrere Male mit Salzeăure gefällt, in kohlcusaurem Ammoniak gelöst und mit Thierkohle gekocht. Das Ammoniaksalz wird durch Chlorbaryum, selbst nach starkem Einengen, nicht gefällt (Ortho- und Parabrombenzoëgäure werden gefällt). Die Säure schmolz bei $137^{\circ}$, sublimirte in flachen Nadeln, die bei $138^{\circ}$ schmolzen. $0.1655 \mathrm{Grm}$. Substanz gaben $0.1530 \mathrm{Grm} . \mathrm{AgBr}=39.3 \mathrm{pCt}$. Brom - die Formel $\mathrm{C}^{7} \mathrm{H}^{3} \mathrm{BrO}^{2}$ verlangt $39.9 \mathrm{pCt}$. Brom. Mit Kalihydrat geschmolzen wurde eine Säure erhalten, die sich mit Eiseuchlorid sturk violett färbte. Beim Zersetzen des Diazoperbromides mit Wasser wurde Salicylsäure erhalten.

Die Metabrombenzoësäure charakterisirt sicb đurch die grosse Löslichkeit des Baryumsalzes. Durch Kochen der Sãure mit Baryumcarbonat erbalten, scheidet sich das Salz beim Verdunsten der Lösung in Warzen ab, die sich sehr leicht in Wasser lösen. 0.6120 Grm. des Salzes, über Schwefelsäure getrocknet, verloren bei $130^{\circ}$ noch 0.0090 Grm. Wasser, - die restirenden $0.6030 \mathrm{Grm}$. gaben $0.2150 \mathrm{Grm}$. $\mathrm{BaO}=29.0 \mathrm{pCt}$.; die Formel $\left(\mathrm{C}^{7} \mathrm{H}^{4} \mathrm{Br}^{2}\right)^{2} \mathrm{Ba}$ verlangt $28.5 \mathrm{pCt}$. Das Baryumsalz der Metabrombenzoësäure, welche aus Chlornitrobenzol gewonneu war, verhielt sich eben so. Das Calciumsalz der Metabrombenzoësäure (aus beiden Quellen) ist weniger löslich; es scheidet sich aus der Lösung in krystallinischeu Krusten ab und enthălt $2 \mathrm{Mol}$. Wasser $\left(\mathrm{C}^{7} \mathrm{H}^{4} \mathrm{BrO}^{2}\right)^{2} \mathrm{Ca}+2 \mathrm{H}^{2} \mathrm{O}$.

Bei einem früheren Versuche, Metabrombenzoësäure aus Anthranilsåure darzustellen, als ich noch nicht die Leicbtlöslichkeit des Baryumsalzes derselben kannte, zersetzte ich das Perbrouid der Diazobenzoësäure aus Anthranilsäure mit Alkohol (96 pCt.) beim Kochen. Aus der Lösung des Natriumsalzes der erhaltenen Bromsăure krystallisirte ein schwerer lösliches Natriumsalz aus, welches von der Flüssigkeit abfiltrirt wurde. Nach einer Wasser- und Natriumbestimmung batte dieses Salz die Formel $\mathrm{C}^{6} \mathrm{H}^{4} \mathrm{Br} . \mathrm{C} \mathrm{O}^{2} \mathrm{Na}+\mathrm{H}^{2} \mathrm{O}$. Die daraus abgeschiedene Bromsäure sublimirte in Nadeln, die bei über $220^{\circ}$ schmolzen; es war also wabrscheinlich Parabrombenzoësäure. Die vom Natriumsalz abfiltrirte Lösung gab eine brombaltige Săure, die bei $139^{\circ}$ scbmolz. Ich balte dafür, dass es die Metabrombenzoësäure war, und dass bei der Entstehung derselben durch Kochen des Diazoperbromides mit Alkohol ein Tbeil derselben zu Parabrombenzoësäure umgesetzt wurde. Derartige theilweise Umsetzungen im status nascendi scheinen nach neueren Beobachtungen hăufiger vorzukommen. Bei der Zersetzung des Diazoperbromids in gelinder Wärme wurde die Bildung von Parabrombenzoësäure neben der Metasäure nicht wahrgenommen. 
Ich babe früher angegeben (Zeitschr. f. Chem. 1869, S. 456), dass aus der rohen Brombenzoësäure (erbalten durch Bromiren von Benzoësäure) eine in Wasser leichter lösliche Brombenzoësäure abgeschieden werden kann, welche ich als Metabrombenzoësäure betrachtete. Da, wie ich daselbst gezeigt, durch Schmelzen der rohen Brombenzoësäure nit Kalihydrat ziemliche Mengen von Salicylsdure erhalten werden (welcbe durch das basische Kalksalz abgescbieden wurde), da mehrere Analysen dieser Säure deo constanten Bromgebalt derselben $(40.0 \mathrm{pCt}$ ) nachwiesen, - so muss ich auch jetzt noch annehmen, dass sich neben der Orthosäure unter gewissen Bedingungen etwas Metabrombenzoëвåure bildet. Den damals angegebenen, auffallend niedrigen Schmelzpunkt der Metabrombenzoësäure, den ich jetzt nacb zwei verschiedenen Darstellungsarten zu $137-138^{\circ}$ gefunden, kann ich nur einer geringen Beimengung von Benzoësäure oder gebromten Benzoësäuren zuschreiben, und glaube, dase es jetzt durch dis Loichtlöslichkeit des Baryumøalzes leicht gelingen dürfte, diese Säure in der rohen Brombenzođsăure nacbzuweisen.

Ueber die Constitution der salpetrigaauren Aether.

Man betrachtet bis jetzt die salpetrigsauren Aether und die Nitrokörper als zwei ganz versehiedene Körperkłassen, auf Grund ihrer verschiedenen Reaction gegen Alkalien und gegen Reductionsmittel. Mendelejeff hat zuerst darauf hingewiesen (siehe diese Berichte III., S. 990), dass diese Trennung eine unbewiesene sei, und dass die Verschiedenbeit der Reaction sich ebenfalls erklären lasse durch die Natur der mit der Gruppe $\mathrm{NO}^{2}$ verbundenen Reste, da die salpetrigsauren Aether nur für die Fettreihe, die Nitroverbindungen dagegen nur für Benzolkörper bekannt sind. Die verachiedene Reactionsfähigkeit der Gruppe $\mathrm{NO}^{2}$ wäre eine ăhnlicbe wie die des Chlors in $\mathrm{C}^{2} \mathrm{H}^{5} \mathrm{Cl}$ und $\mathrm{C}^{6} \mathrm{H}^{3} \mathrm{Cl}$, oder in den gecblorten Toluolen $\mathrm{C}^{6} \mathrm{H}^{5} \mathrm{CH}^{2} \mathrm{Cl}$ und $\mathrm{C}^{6} \mathrm{H}^{4} \mathrm{Cl} . \mathrm{CH}^{3}$. Es scheint mir, dass durch die ron mir gefundene Reaction von Cyankalium auf Nitrokörper diese Ansicht bestätigt wird. Die Nitro-Grappe scheidet sich hierbei als salpetrigsaures Kali $\mathrm{KNO} \mathrm{O}^{2}$ aus, was dafür spricht, dass bei beiden Körpern die Grappe $\mathrm{NO}^{2}$ dieselbe Structur besitzt. Wenn diese Ansicht richtig ist, so war zu erwarten, dass das Cyankalium anf die salpetrigsauren Aether eben so wirken würde, wie auf die Chlor- und Bromnitrobenzole. Es scheint dieses in der That der Fall za sein. Beim Erhitzen von Nitroäthan (salpetrigsaurem Aetbyläther) mit Cyankalium und Alkohol in zugeschmolzenen Röhren finden ganz die angegebenen Erscheinungen statt. Es scheidet sich salpetrigsaures Kali aus und beim Kochen mit Kali entwickelt sich yiel Ammonials. Aus der mit Schwefelsäure übersāttigten Lösung extrabirt Aether eine Süure, die stark buttersüureartig roch. Es gelang mir aber noch nicht 
dies erwartete Propionsïure in zur Analyse genūgender Menge zo gowinnen, da ich die richtige Reactionstemperatur noch nicht festgestellt, - häufig explodiren die Röbren. Ebenso verbielt es sich mit Nitropentan (salpetrigsaurem Amyl).

Wenn in den salpetrigsauren Aethern und in den Nitrokörpern die Gruppe $\mathrm{NO}^{2}$ dieselbe Structur besitzt, 80 muss jedenfalls angenommen werden, dass die Bindung von $\mathrm{NO}^{2}$ mit dew Koblenstoff durch das Stickstoffatom vermittelt wird, wobei nocb dabingestellt bleibt. ob der Stickstoff hier drei- oder fünfwerthig wirkt. Mlsdann muss auch im salpetrigsanren Kali das Metall mit dem Stickstoff verbunden sein. Es stellt dies bei näherer Betrachtung nichts besuuder» Abnormes dar, da erstens das salpetrigsaure Silbersalz $\mathrm{AgNO}^{2}$ (wie auch Bleisalz) mit dem Chlorsilber $\mathrm{AgCl}$ in der Schwerlöslichkeit einige Aehnlichkeit zeigt, und da zweitens wir ja anch in den schwefligsauren Salzen die Bindung eines Metallatoms mit Schwefel annehmen $\mathrm{KSO}^{2} \mathrm{OK}$, - wie ich dies ausführlicher in meinem "Lehrbuch der organischen Chemie, begründet auf der Constitutionstheorie, 1870 an den Aethern der schwefeligen Säure (Sulfosäuren) entwickelt.

Es fragt sich ferner, wie erklärt sich bei dieser Anschauung das Verbalten der wahren Nitrofettkörper, des Nitrofroms, Nitroformens, Chlorpikrins, welche mit Kalilauge nicbt reagiren. Wir wissen aber, dass das Chlorpikrin mit Natriumäthylat die Nitrogruppe als salpetrigsaures Kali ausscheidet (Basset, Zeitschr. f. Chem. 1864, 281), wobei der vierbasische Kohlensäureäther entsteht:

$\mathrm{C}\left(\mathrm{NO}^{2}\right) \mathrm{Cl}^{3}+4 \mathrm{C}^{2} \mathrm{H}^{5} \cdot \mathrm{ONa}=\mathrm{NaNO}^{2}+\mathrm{C}\left(\mathrm{O} . \mathrm{C}^{2} \mathrm{H}^{5}\right)^{4}+3 \mathrm{NaCl}$.

Für das Nitroform und Nitroformen $\mathrm{C}\left(\mathrm{NO}^{2}\right)^{4}$ ist meines Wissens diese Reaction noch nicht versucht; die Beständigkeit der Nitrogruppen in denselben ist aber die nämliche, wie die des Chlors im Chloroform $\mathrm{CHCl}^{3}$, welches durch wässeriges Kali und Silberoxyd nicht ausgetauscht wird, während $\mathrm{CH}^{3} \mathrm{Cl}$ leicht reagirt; - der weitere Eintritt von negativen Gruppen verbindert die Reactionsfähigkeit der ersten.

Jedenfalls wäre es wichtig, durch weitere Versuche diese Anschauung za prüfen.

St. Petersburg, April 1871.

138. Adolf Schröder: Untersuchungen über den Valeraldehyd. (II. Theil.)

(Aus dem Berl. Univ.-Lab. LXXXI; vorgetr. von Hrn. A. W. H of mann.)

$$
\text { Valeraldin. }
$$

Durch Ueberleiten trockenen Ammoniakgases über Sulfovaleraldehyd entsteht Valeraldin,

$$
3 \mathrm{C}_{5} \mathrm{H}_{10} \mathrm{~S}+2 \mathrm{NH}_{3}=\mathrm{C}_{15} \mathrm{H}_{31} \mathrm{NS}_{2}+\mathrm{NH}_{4} \text {, HS. }
$$

